\title{
Conducting a pre-consent process for clinical trials: Promoting a more-true informed consent
}

\author{
Wong $\mathrm{LX}^{1,2 *}$, WongJC ${ }^{3}$, Chee B ${ }^{4}$, Bloom GM${ }^{1}$, Sharma MJ ${ }^{5}$, Liang G6 and Park JM7 \\ ${ }^{1}$ Department of Clinical Research and Leadership, The George Washington University, School of Medicine and Health Sciences, USA \\ ${ }^{2}$ Department of Hematology and Oncology, Gastrointestinal Oncology, University of California, San Francisco, USA \\ ${ }^{3}$ Department of Nursing, University of San Francisco - School of Nursing and Health Professions, USA \\ ${ }^{4}$ Department of Primary Care, Touro University College of Osteopathic Medicine - California, USA \\ ${ }^{5}$ Department of Pulmonary, Critical Care, Allergy and Sleep Medicine, Interstitial Lung Disease, University of California, San Francisco, USA \\ ${ }^{6}$ Department of Law, Santa Clara University - School of Law, USA \\ ${ }^{7}$ Department of Medical Education, Rush University Medical College, USA
}

\begin{abstract}
We intend for this manuscript to propose the paradigm shift in which the informed consent process starts at the clinical visit to the point of first pre-consent contact. This includes early trust-building communication and incorporating key stakeholders at pre-consent stages in this patient-centric process. The goal is to provide a more seamless experience for the participant through an improved pre-consent process, allowing ample time for discussion of available options, decision-making, and potentially more informed decision.
\end{abstract}

\section{Introduction}

It is ultimately the responsibility of clinical research healthcare providers to improve the process to insure a true informed consent for all potential participants.

In practice, the informed consent process between a participant and an investigator tends to start during a consent visit. Usually a physician, the investigator will begin by reviewing therapeutic treatment options, with oftentimes a single investigative, interventional therapeutic option [1]. In this setting, during a short consultation, there is a sense of pressures mounting and the participant is likely to feel obligated to choose the therapeutic option [2]. Additionally, a participant may find it difficult to fully comprehend the nature of a complex investigative clinical trial. There may be a complicated scientific rationale and participants may experience information overload or inability to concentrate or retain information in a high-stress environment [3]. Moreover, in an unfamiliar setting, a participant may be asked to assess the potential changes in quality of life, relative benefit due to the treatment option, logistics, and costs [4].

At the time of this writing, ClinicalTrials.gov lists over a third of a million studies active in all 50 states and in 210 countries [5]. The explosion of clinical research conducted globally is underscored when considering just over one thousand studies were active in the year 2000. With an increase of active investigative therapeutic clinical trials, there are increases in the need to obtain informed consent. This means there are more clinic visits and there are more modes of transmission of information. Logistically, financially, ethically, and spiritually there have been increases in complexity. As a result, there is a need for added sensitivity when obtaining informed consent in clinical research [6].

\section{Discussion}

\section{Informed consent regulatory guidelines}

One of the hallmark protections established for participants in clinical trials is the informed consent process [7]. At the core of the Nuremberg Code, established in 1947 [8], is a set of ethical guidelines that are accepted on an international basis. Research investigators are required to encourage effective and continual communication between themselves and the participant grounded in the intent to promote voluntary participation in clinical trials, a trusting relationship, and respect of persons. The essential voluntary aspects of being informed are capacity to consent, freedom from coercion, and understanding of the risks and benefits. Adherence to the Code of Federal Conduct provides protections for participants in clinical trials.

Countries and international working bodies have built upon the foundation of the Nuremberg Code to reinforce the protection and safety of participants in clinical trials. The 1949 International Code of Medical Ethics of the World Medical Assembly, including the Declaration of Geneva, outlines the responsibilities of healthcare providers, specifically physicians, for "providing competent medical

${ }^{\star}$ Correspondence to: Louis Wong, MS, PhD Student, Translational Health Sciences, Clinical and Translational Research, The George Washington University, School of Medicine and Health Sciences, USA, E-mail: louiswong415@gwmail.gwu.edu

Key words: capacity to consent, clinical trials, disclosure of information, informed consent process, participant, pre-consent, patient-centric, protection, safety, trust, understanding, voluntariness

Received: July 27, 2020; Accepted: August 21, 2020; Published: August 24, 2020 
service in full technical and moral independence, with compassion and respect for human dignity" and to "bear in mind the obligation of preserving human life." The 1964 Declaration of Helsinki provides guidance for physicians conducting clinical research with a focus on researchers' roles and responsibilities to protect human subjects, including the process and elements of obtaining informed consent [9]. The 1974 Belmont Report emphasizes the three ethical principles of respect for persons, beneficence, and justice. These three key principles are applied to research through application of informed consent, assessment of risks and benefits, and selection of subjects. The Belmont Report and these other guidelines and regulations influence current standards and set the foundation for clinical research across the globe $[10,11]$.

\section{Elements in the informed consent}

The fundamental format of the informed consent, as well as the conversations to obtain consent, should be entrenched in four quintessential elements: complete disclosure of information, understanding, voluntariness, and capacity to consent (45 CFR $\$$ 46.116, 2018).

\section{Disclosure of information}

The goal of information disclosure is to allow the potential participant to identify relevant information towards making an informed decision. Healthcare providers are shifting to individualized consent disclosure based on the individual's values and needs rather than presenting exhaustive one-size-fits-all information [12]. Individualized consent disclosure includes accurate information about the research aim, study duration, likely benefits and potential risks, alternative treatment options, and the right to withdraw from the trial [13].

\section{Understanding}

The objective of understanding is to attain comprehension of the risks, benefits, and nature of the procedure and the principles directly affecting care [14]. This includes a review of the investigational drugs, study visit requirements, and duration of the study.

\section{Voluntariness}

The goals of voluntariness of a consent highlights the decision making of an individual without coercion or the undue influence by someone else [15]. The informed consent is not a binding contract that requires a participant to commit to a single treatment option because the voluntary informed consent process is dynamic and ongoing. The aim is to consistently inform the participant about any changes from start to end of the clinical trial [16].

\section{Capacity to consent}

The capacity to consent is decisional capacity, or the ability to understand, appreciate, and reason when given information in order to make a choice. This involves ability to consider the risks and benefits of the procedure with personal life goals. Institutions often use the MacArthur Competence Assessment Tool for Clinical Research (MacCAT-CR) to measure a potential participant's capacity to consent for research participation [17].

\section{Best Practices: Early intervention for a pre-consent process}

The traditional procedure for the informed consent process starts at the first consultation, which may be weeks after the first contact. We suggest a shift in the paradigm to start the consent process before the first initial contact with the participant, particularly before a clinical visit [18]. It is challenging for the typical potential participant to fully comprehend all the components necessary for informed consent only moments after consent is introduced in an unfamiliar and stressproducing environment. It is in the participant's best interest to start communication earlier in the process rather than at a scheduled consent visit. The consent process should begin at the moment a potential participant is identified in the queue. Proper preparation made ahead of time can provide the potential participant with ample time to read, understand, and prepare questions in anticipation for the visit.

This early intervention for starting the informed consent process can streamline the consent process across clinical and research settings for obtaining true informed consent. The practice can make a great positive impact on participant care by decreasing stress and uncertainty. Early intervention can increase trust and transparency between the participant and investigator in investigational clinical trials. This approach can save clinical time because of an increase in the likelihood of participants providing consent at the initial clinic visit rather than scheduling additional visits for clarification prior to the consent. The pre-consent process should be added to the planning timeline for routine scheduling of institutional consenting workflow. From a clinical research coordinator perspective, the basis of clinical research is ensuring the participant fully understands the elements of the study prior to consenting. This practice streamlines the process and would facilitate the scheduling of clinical trial events more easily. The coordinator would be involved with the participant earlier in the process to better establish trust and transparency. Involving the key stakeholders inclusive of the participant and family in conjunction with the study team of the physician, nurses, research coordinators, and research investigators provides for a more well-rounded crossfunctional level of care.

Effective communication is essential to a safe practice. Providing participants with relevant information prior to obtaining informed consent allows ample time to process the large amounts of information. Through transparency and empathy, we continue to serve as advocates and empower participants to make clinical decisions. A patient-centered approach allows participants to maintain control over the process and to make an educated decision. This perspective with its participant advocacy focus encourages the participants to better understand the issues and factors. They have more time to go through the process to decide if they would like to participate in the clinical trial [19-22].

This pre-consent practice would lead to the next step in the informed consent process with the principal investigator. The traditional procedures would be bolstered by increased trust and transparency when a more aware potential participant can come to the meeting prepared to make a truly informed decision to give consent.

\section{Proposed pre-consent workflow}

The following practice procedure is intended to provide some guidance for multidisciplinary clinical trial research teams. It is prepared with an awareness of international law and regulations to maximize participant protection and safety while obtaining a true informed consent.

\section{Initial pre-consent contact}

1. The healthcare provider team will identify available options for the potential participant from an initial screening of future scheduled treatment appointments. 
2. Contact the potential participant to determine potential interest and to confirm contact information.

3. Transmit informed consent documents and information in the participant's preference (i.e. electronic documents via electronicmail, physical documents delivered by mail). Provide unsignable, sample informed consent forms (ICFs), Health Insurance Portability and Accountability Act (HIPAA) disclosures, and Patient's Bill of Rights (BOR). If applicable, send in appropriate languages and formats. Include a single-paged fast fact sheet with an overview of study information, possible risks and benefits, and inclusion and exclusion criteria. List team contact information for the trial.

4. Coordinate and schedule a future call for discussion and to answer questions with potential participant, research coordinator, nurse, and/or family members.

\section{Pre-Consent}

\section{Call}

1. Introduce key healthcare team members.

2. Answer questions and introduce relevant topics within their appropriate professional qualifications.

3. Schedule call for next session to obtain informed consent. Provide contact email for follow-up questions.

\section{Research agenda}

Promoting patient understanding to facilitate a more-true informed consent calls for a change in our approach by shifting the paradigm in which the informed consent process starts and by incorporating key stakeholders at early stages in the patient-centric process. These suggested procedures lay the fundamental foundations to evaluate the efficacy of these practices. Research could measure the levels of trust and the extent of understanding these participants have when in a clinical research setting. Future studies could involve a randomized control trial (RCT) within an investigative study where one of two cohorts would go through the full pre-consent process whereas the other cohort would partake in the traditional informed consent process on the first day of treatment consultation.

We are aware of no studies investigating this process. An evidencebased study would be valuable and necessary to investigate this particular approach as a practical method to promote understanding towards achieving a more-true informed consent.

\section{Conclusion}

Additionally, these innovative concepts may spur working groups and task committees to adopt a pre-consent procedure with development of a workflow plan to suit their own institutional practices and guidelines. Institutions can collaborate cross-functionally creating cooperative multi-disciplinary teams.

\section{Conflicts of interest}

The authors have no conflict of interest to report.

\section{Acknowledgments}

We thank the following individual for their academic support and guidance in clinical research and the informed consent process: Samar Nasser, PhD, MS, MPH, The George Washington University and Andrew H. Ko, MD, University of California, San Francisco.

\section{References}

1. Hall DE (2012) Informed consent for clinical treatment. CMAJ 184: 533-540.

2. Say RE (2003) The importance of patient preferences in treatment decisions--challenges for doctors. BMJ 327: 542-545. [Crossref]

3. Dankar FK (2019) Informed consent in biomedical research. Computational and Structural Biotechnology Journal 17: 463-474. [Crossref]

4. Spatz ES (2016) The new era of informed consent: Getting to a reasonable-patient standard through shared decision making. JAMA 315: 2063-2064. [Crossref]

5. United States National Institutes of Health (NIH). (2020, April 21). Trends, Charts, and Maps. Retrieved from https://clinicaltrials.gov/ct2/resources/trends.

6. Gupta UC (2013) Informed consent in clinical research: Revisiting few concepts and areas. Perspectives in Clinical Research 4: 26-32.

7. Nijhawan LP (2013) Informed consent: Issues and challenges. Journal of Advanced Pharmaceutical Technology and Research 4: 134-140. [Crossref]

8. Annas GJ (2018) Beyond Nazi war crimes experiments: The voluntary consent requirement of the nuremberg code at 70. Am J Public Health 108: 42-46. [Crossref]

9. Striefel S (2001) Appl Psychophysiol Biofeedback 26: 39-59; discussion 67-71. Ethical research issues: going beyond the Declaration of Helsinki.

10. Breault JL (2006) Protecting human research subjects: the past defines the future. The Ochsner Journal 6: 15-20. [Crossref]

11. Fischer BA 4th (2006) A summary of important documents in the field of research ethics. Schizophrenia Bulletin 32: 69-80.

12. Siegal G (2012) Personalized disclosure by information-on-demand: attending to patients' needs in the informed consent process. The Journal of Law, Medicine \& Ethics 40: 359-367.

13. Mexas F (2014) Understanding and retention of trial-related information among participants in a clinical trial after completing the informed consent process. Clinical Trials 11: 70-76. [Crossref]

14. Cameron P (2013) A comparison of patient knowledge of clinical trials and trialist priorities. Current Oncology (Toronto, Ont.) 20: e193-e205.

15. Appelbaum PS (2009) Voluntariness of consent to research- A conceptual model. Hastings Center Report 39: 30-39. [Crossref]

16. Jefferson AL (2008) Decisional capacity for research participation in individuals with mild cognitive impairment Journal of the American Geriatrics Society 56: 1236-1243. [Crossref]

17. Wong LX, Bloom GM, Chee B (2020) The complex maze of the informed consent process: Helping to improve comprehension in clinical trial participants with Alzheimer's disease. J Alzheimers Dis Rep 4: 161-164.

18. Department of Health, Education, and Welfare (1974, July) The Belmont Report Ethical principles and guidelines for the protection of human subjects of research Retrieved April 26, 2020, from https://www.hhs.gov/ohrp/regulations-and-policy/ belmont-report/read-the-belmont-report/index.html

19. General requirements for informed consent, 45 CFR $\S 46.116$ (2018).

20. Gossman W, Thornton I, Hipskind JE (2020) Informed consent. [Updated $2020 \mathrm{Feb}$ 18]. In: StatPearls [Internet]. Treasure Island (FL): StatPearls Publishing; 2020 Jan-. Available from: https://www.ncbi.nlm.nih.gov/books/NBK430827/.

21. World Medical Association (1949, October) International code of medical ethics of the world medical assembly, including the declaration of Geneva. Retrieved April 26 , 2020, from https://history.nih.gov/research/downloads/icme.pdf.

22. World Medical Association (1964, June) Declaration of Helsinki. Retrieved April 26 , 2020 from https://history.nih.gov/research/downloads/helsinki.pdf.

Copyright: (C2020 Wong LX. This is an open-access article distributed under the terms of the Creative Commons Attribution License, which permits unrestricted use, distribution, and reproduction in any medium, provided the original author and source are credited. 